\section{(d) \\ CrossMark}

\title{
The independent contribution of Pseudomonas aeruginosa infection to long-term clinical outcomes in bronchiectasis
}

\author{
David Araújo ${ }^{1}$, Michal Shteinberg ${ }^{2}$, Stefano Aliberti $\mathbb{1}^{3,4}$, Pieter C. Goeminne ${ }^{5,6}$, \\ Adam T. Hill ${ }^{7}$, Thomas C. Fardon ${ }^{8}$, Dusanka Obradovic ${ }^{9}$, Glenda Stone ${ }^{10}$, \\ Marion Trautmann ${ }^{10}$, Angela Davis ${ }^{10}$, Katerina Dimakou ${ }^{11}$, Eva Polverino ${ }^{12}$, \\ Anthony De Soyza (13 ${ }^{13,14}$, Melissa J. McDonnell ${ }^{14,15}$ and James D. Chalmers ${ }^{8}$
}

@ERSpublications

Frequent exacerbations are the key determinants of long-term outcome in patients with chronic Pseudomonas aeruginosa infection http://ow.ly/WjPZ30h67rL

Cite this article as: Araújo D, Shteinberg M, Aliberti S, et al. The independent contribution of Pseudomonas aeruginosa infection to long-term clinical outcomes in bronchiectasis. Eur Respir J 2018; 51: 1701953 [https://doi.org/10.1183/13993003.01953-2017].

ABSTRACT Pseudomonas aeruginosa is responsible for chronic infection in many bronchiectasis patients but it is not known whether it is associated with worse clinical outcomes independent of the underlying severity of disease.

This study analysed data from 2596 bronchiectasis patients included from 10 different bronchiectasis clinical centres across Europe and Israel, with a 5-year follow-up period. Prevalence of P. aeruginosa chronic infection and its independent impact on exacerbations, hospitalisations, quality of life and mortality was assessed.

The prevalence of $P$. aeruginosa chronic infection was $15.0 \%(\mathrm{n}=389)$. P. aeruginosa was associated with a higher mortality in a univariate analysis (hazard ratio (HR) 2.02; 95\% (confidence interval) CI 1.53-2.66; $\mathrm{p}<0.0001$ ) but an independent impact on mortality was not found in a multivariate analysis (HR 0.98; 95\% CI 0.70-1.36; $\mathrm{p}=0.89$ ). $P$. aeruginosa was independently associated with increased mortality only in patients with frequent exacerbations (two or more per year) (HR 2.03; 95\% CI 1.36-3.03; p=0.001). An independent association with worse quality of life of 7.46 points (95\% CI 2.93-12.00; $\mathrm{p}=0.001$ ) was found in a multivariable linear regression. $P$. aeruginosa was therefore found to be independently associated with exacerbation frequency, hospital admissions and worse quality of life. Mortality was increased in patients with $P$. aeruginosa particularly in the presence of frequent exacerbations.

Published online Feb 01 2018; republished Feb 062018 with amendments to the authors' affiliation details.

This article has supplementary material available from erj.ersjournals.com

Received: Sept 252017 | Accepted after revision: Nov 132017

Support statement: This study was supported by a research grant from Grifols. Funding information for this article has been deposited with the Crossref Funder Registry.

Conflict of interest: Disclosures can be found alongside this article at erj.ersjournals.com

Copyright @ERS 2018 
Affiliations: ${ }^{1}$ Pulmonology Dept of São João Hospital Center, Porto, Portugal. ${ }^{2}$ Pulmonary Institute and CF Center, Carmel Medical Center, Haifa, Israel. ${ }^{3}$ Dept of Pathophysiology and Transplantation, University of Milan, Milan, Italy. ${ }^{4}$ Internal Medicine Dept, Respiratory Unit and Cystic Fibrosis Adult Center, Fondazione IRCCS Ca' Granda Ospedale Maggiore Policlinico, Milan, Italy. ${ }^{5}$ Respiratory Medicine, University Hospital Gasthuisberg, Leuven, Belgium. ${ }^{6}$ Respiratory Disease, AZ Nikolaas, Sint-Niklaas, Belgium. ${ }^{7}$ Royal Infirmary of Edinburgh and University of Edinburgh, Edinburgh, UK. ${ }^{8}$ Scottish Centre for Respiratory Research, University of Dundee, Dundee, UK. ${ }^{9}$ Institute for Pulmonary Diseases of Vojvodina Sremska Kamenica and Faculty of Medicine, University of Novi Sad, Novi Sad, Serbia. ${ }^{10}$ Grifols Inc., Research Triangle, NC, USA. ${ }^{11} 5$ th Dept of Pulmonary Medicine, "Sotiria" Chest Diseases Hospital, Athens, Greece. ${ }^{12}$ Thorax Institute, Institute of Biomedical Research August Pi i Sunyer (IDIBAPS), Ciberes University of Barcelona, Vall d'Hebron Research Institute VHIR, Hospital Vall D'Hebron, Respiratory Disease Dept, Barcelona, Spain. ${ }^{13}$ Adult Bronchiectasis Service and Sir William Leech Centre for Lung Research, Freeman Hospital, Newcastle upon Tyne Hospitals NHS Foundation Trust, Heaton, UK. ${ }^{14}$ Institute of Cellular Medicine, Newcastle University, Newcastle upon Tyne, UK. ${ }^{15}$ Dept of Respiratory Medicine, Galway University Hospitals, Galway, Ireland.

Correspondence: James D. Chalmers, Scottish Centre for Respiratory Research, University of Dundee, Ninewells Hospital and Medical School, Dundee, DD1 9SY, UK. E-mail: jchalmersdadundee.ac.uk

\section{Introduction}

Bronchiectasis is a chronic and progressive respiratory disease characterised by permanent dilation of bronchi, impairment of mucus clearance, chronic cough and sputum production, and an increased frequency of respiratory infections [1]. Without effective mucociliary and innate antimicrobial defences, there is a higher risk of chronic bacterial infection of the airways, which can lead to an increase in airway inflammation and structural damage $[2,3]$.

Pseudomonas aeruginosa is one of the most important organisms causing chronic infection in bronchiectasis, because of its capacity to maintain virulence despite antibiotic therapies by forming biofilms and easily developing antimicrobial resistance [4-6]. P. aeruginosa in cystic fibrosis (CF) bronchiectasis is associated with a steeper decrease in lung function and increased morbidity and mortality [7]. Its presence in bronchiectasis seems to be linked with a more severe disease, but whether there is a direct effect on disease progression or whether $P$. aeruginosa is just an indicator of existing clinical severity remains a topic of discussion [8-10].

In a recent meta-analysis, $P$. aeruginosa chronic infection was related to a threefold increase in mortality, as well as a higher risk of hospital admissions and increased exacerbation frequency [11]. This study however, was not able to take into account the multiple confounders that are usually present in more severe bronchiectasis patients and that could play a role in mortality, such as forced expiratory volume in $1 \mathrm{~s}$ (FEV1), comorbidities or severe exacerbations. Moreover, there was some heterogeneity in terms of chronic infection definition and measured parameters across the different studies [11]. To definitively answer whether $P$. aeruginosa directly drives disease progression and mortality in bronchiectasis would require a longitudinal study able to identify the point at which $P$. aeruginosa infection first occurs and to compare morbidity before and after acquisition. As such studies are not currently available, an alternative method is to utilise multivariable modelling, which allows the estimation of the independent contribution of $P$. aeruginosa after removing the effects of confounding variables.

The rate of $P$. aeruginosa chronic infection in bronchiectasis patients varies among the studies, between 9$31 \%$, and its prevalence in a large, multicentre population from several different countries is yet to be assessed [12-18].

The aim of this study was to assess the burden of disease in P. aeruginosa chronic infection and its independent impact in terms of patient outcomes.

\section{Methods}

This study analysed data from bronchiectasis patients enrolled in 10 different bronchiectasis clinical centres across Europe (including Israel as an EU affiliated country) (Dundee, Edinburgh, Newcastle - UK; Haifa Israel; Galway - Ireland; Leuven - Belgium; Athens - Greece; Monza - Italy; Barcelona - Spain; Vojvodina Serbia) between 2007 and 2013. Consecutive patients aged $\geqslant 18$ years with a diagnosis of bronchiectasis, made by a specialist physician, based on a high-resolution computed tomography scan and with a compatible clinical history (defined by the presence of respiratory symptoms attributable to bronchiectasis) were enrolled. Patients had to be free from antibiotic treatment for exacerbation for 4 weeks prior to enrolment and initial data collection. Patients with CF or traction bronchiectasis because of pulmonary fibrosis were excluded, and also patients with active nontuberculous mycobacterial (NTM) disease. The local ethics committee or institutional review board approved the data collection at each site. Diagnostic work-up and assessment were made following algorithms consistent with the 2010 British Thoracic Society (BTS) guidelines [19]. 
Identification of microorganisms and susceptibility testing were performed according to standard methods [20]. Chronic infection was defined by isolation of the same pathogen in two or more cultures, at least 3 months apart in a 12-month period [19].

Demographics, previous medical history, comorbidities as well as radiological, laboratory and microbiological findings were obtained from a combination of direct patient history, medical records and laboratory information recorded at each site. Quality of life (QoL) was measured by the St George's Respiratory Questionnaire (SGRQ), and severity of disease was evaluated by the Bronchiectasis Severity Index (BSI) [21]. There was a follow-up period of 5 years, in which exacerbations and hospitalisations were recorded, following BTS guidelines definitions, and mortality was assessed.

\section{Statistical analysis}

Simple descriptive statistics of mean with standard deviation (SD) were used for continuous parametric data, median with $25-75 \%$ interquartile range (IQR) for continuous nonparametric data, and frequencies and percentages for categorical data. Subgroup comparisons were performed using the unpaired t-test, Mann-Witney U-Test or Chi-squared test, depending on data distribution.

Kaplan-Meier curves were used to illustrate survival data. For multivariable mortality analysis, Cox proportional hazard regression analysis was performed to estimate hazard ratios (HRs) and their 95\% confidence intervals (CI). Variables included in the model were those determined by the investigators to be clinically significant in impacting on mortality. In a sensitivity analysis, changes in prophylactic antibiotic therapy during the study were examined as a confounder in a Cox proportional hazard regression with treatment as a time-varying covariate.

A multiple linear regression model was applied to determine the variables with an independent impact on QoL. To check the suitability of our model, we fitted a generalised linear model (GLM) with quasibinomial errors and a logit link. The signs and significance of every estimated parameter in this matched its equivalent in the linear model. Estimates of the change in fitted values, from the GLM, on stepping one unit away from the centre of the dataset (the medians of all numerical values) in each direction all fell within the 95\% CI around the betas in the linear model. However, while the model results are very similar, the terms within the generalised linear model are difficult to interpret. We have, therefore, described the results of the linear model.

All analyses were performed using SPSS version 21 (SPSS, Chicago, IL, USA) for Windows platform and Graph Pad Prism Version 5 (Graph Pad Software, San Diego, CA, USA).

\section{Results}

\section{Patient characteristics}

The study population was composed of 2596 patients. Individual inclusion per site was 608 in Edinburgh, 504 in Dundee, 116 in Newcastle, 280 in Galway, 190 in Leuven, 113 in Vojvodina, 88 in Haifa, 159 in Athens, 198 in Barcelona and 340 in Monza.

The patient characteristics are described in detail in table 1.

The median age was 67 years (IQR 57-74 years) and there was a female predominance $(61.1 \%, \mathrm{n}=1586)$. In terms of comorbidities, the highest reported conditions were ischaemic heart disease, $17.5 \%(\mathrm{n}=453)$, chronic obstructive pulmonary disease (COPD), 16.6\% $(\mathrm{n}=431)$, gastro-oesophageal reflux disease (GORD), 15.2\% $(\mathrm{n}=394)$ and diabetes, $10.0 \%(\mathrm{n}=260)$. The median BSI score was 6 (IQR 4-10) with a homogeneous distribution between the severity groups: mild (0-4) (29.0\%, $\mathrm{n}=753)$, moderate (5-8) $(35.7 \%, n=927)$, severe $(\geqslant 9)(35.3 \%, n=916)$. Patients had a median of 41.2 points in the SGRQ (IQR 24.5-59.6). Most patients were classified as idiopathic (42\%), with post-infective aetiology (17\%), COPD (9\%), asthma (6\%), connective tissue diseases (6\%) and allergic bronchopulmonary aspergillosis (5\%) also being common in this cohort.

At the time of enrolment, the median number of exacerbations in the previous year was two (IQR $0-3$ ); however, $37.2 \%(n=966)$ had three or more exacerbations over the same period, and $25.9 \%(n=672)$ had at least one hospitalisation in the previous year.

\section{Pseudomonas aeruginosa chronic infection prevalence}

Half of the study population had a chronic respiratory infection with at least one pathogen $(50.4 \%$, $\mathrm{n}=1307)$. The most common were Haemophilus influenzae $(21.9 \%, 95 \%$ CI $20.4-23.6 \%, \mathrm{n}=569)$ and P. aeruginosa $(15.0 \%, 95 \%$ CI 13.7-16.4\%, $\mathrm{n}=389)$. Rates of $P$. aeruginosa infection varied across different European centres, from the lowest in Serbia 0.9\% (95\% CI 0.2-4.8\%), Belgium 8.4\% (95\% CI 5.3-13.2\%), Italy $12.1 \%$ (95\% CI 9.0-15.9\%), UK 12.5\% (95\% CI 10.7-14.4\%) and Ireland 13.9\% (95\% CI 10.4-18.5\%) 


\begin{tabular}{|c|c|}
\hline Patients & $2596(100 \%)$ \\
\hline \multicolumn{2}{|l|}{ Demographics } \\
\hline Age years & $67(57-74)$ \\
\hline$\geqslant 65$ years old & $1395(53.7 \%)$ \\
\hline Male & $1010(38.9 \%)$ \\
\hline $\mathrm{BMI} \mathrm{kg} \cdot \mathrm{m}^{-2}$ & $24.8(21.8-28.1)$ \\
\hline Smokers and ex-smokers & $990(38.1 \%)$ \\
\hline \multicolumn{2}{|l|}{ Comorbidities } \\
\hline Ischaemic heart disease & $453(17.5 \%)$ \\
\hline Stroke & $152(5.9 \%)$ \\
\hline Diabetes & $260(10.0 \%)$ \\
\hline Liver disease & $41(1.6 \%)$ \\
\hline Chronic renal failure & $154(5.9 \%)$ \\
\hline COPD & $431(16.6 \%)$ \\
\hline Asthma & $226(8.7 \%)$ \\
\hline Connective tissue disease & $210(8.1 \%)$ \\
\hline Neurological disease & $68(2.6 \%)$ \\
\hline Osteoporosis & $192(7.4 \%)$ \\
\hline GORD & $394(15.2 \%)$ \\
\hline Haematological malignancy & $33(1.3 \%)$ \\
\hline Solid tumour & $164(6.3 \%)$ \\
\hline \multicolumn{2}{|l|}{ Functional status } \\
\hline FEV $1 \%$ predicted & $73.8(54.0-92.1)$ \\
\hline FEV $1<50 \%$ predicted & $502(19.3 \%)$ \\
\hline \multicolumn{2}{|l|}{ Clinical status } \\
\hline Number of exacerbations in the previous year & $2(0-3)$ \\
\hline Three or more exacerbations per year & $966(37.2 \%)$ \\
\hline At least one hospitalisation in the previous year & $672(25.9 \%)$ \\
\hline \multicolumn{2}{|l|}{ Disease severity } \\
\hline BSI score & $6(4-10)$ \\
\hline Mild BSI score ${ }^{\#}$ & $753(29.0 \%)$ \\
\hline Moderate BSI score ${ }^{\pi}$ & $927(35.7 \%)$ \\
\hline Severe BSI score ${ }^{+}$ & $916(35.3 \%)$ \\
\hline \multicolumn{2}{|l|}{ Quality of life } \\
\hline$S G R Q^{\S}$ & $41.2(24.5-59.6)$ \\
\hline
\end{tabular}

Data are presented as median (interquartile range) unless otherwise stated. BMI: body mass index; COPD: chronic obstructive pulmonary disease; GORD: gastro-oesophageal reflux disease; FEV1: forced expiratory volume in $1 \mathrm{~s}$; BSI: Bronchiectasis Severity Index; SGRQ: St George's Respiratory Questionnaire. \#: BSI 0-4; ๆ: $\mathrm{BSI} 5-8 ;{ }^{+}: \mathrm{BSI}>9 ;^{\S}: \mathrm{n}=987$.

to the highest rates in Spain 21.2\% (95\% CI 16.1-27.4\%), Greece 36.5\% (95\% CI 29.4-44.2\%) and Israel 44.3\% (95\% CI 34.4-54.7\%). Active NTM was excluded, but NTM isolation as baseline was reported in 46 subjects.

\section{Patient characteristics according to chronic infection status}

Patients with $P$. aeruginosa were slightly older (median (IQR), 70 years (59-77 years) versus 67 years (5774 years) versus 66 years $(57-75$ years $), \mathrm{p}<0.001)$ and had a lower $\mathrm{FEV}_{1} \%(57.9 \%$ versus $69.2 \%$ versus $77.0 \%, \mathrm{p}<0.001)$ than patients chronically infected with other pathogens or those not chronically infected respectively (table 3 ). They had more major cardiovascular comorbidities such as ischaemic heart disease and stroke. They had also more exacerbations on the previous year (median (IQR) 3 (2-4) versus 2 (1-3) versus $1(0-2), \mathrm{p}<0.001)$ and a higher percentage of patients with at least one hospital admission in the previous year $(59.9 \%$ versus $31.9 \%$ versus $17.6 \%, \mathrm{p}<0.000)$. The median BSI was higher in the P. aeruginosa group (median (IQR) 13 (10-17) versus 8 (5-13) versus 5 (3-8), p<0.001). Data are presented in table 2.

\section{Mortality analysis}

There were 281 deaths during the follow-up period, which accounted for $10.8 \%$ (95\% CI 9.7-12.1\%) of the total study population. There were 73 deaths in 389 patients (18.8\%) in the P. aeruginosa chronic infection group, 75 in $918(8.2 \%)$ in the other pathogens chronic infection group and 133 in 1289 (10.3\%) in patients with no chronic infection ( $\mathrm{p}<0.0001$ comparing all three groups). 
TABLE 2 Differences between patients with chronic Pseudomonas aeruginosa infection, chronic infection by other pathogens and no chronic infection

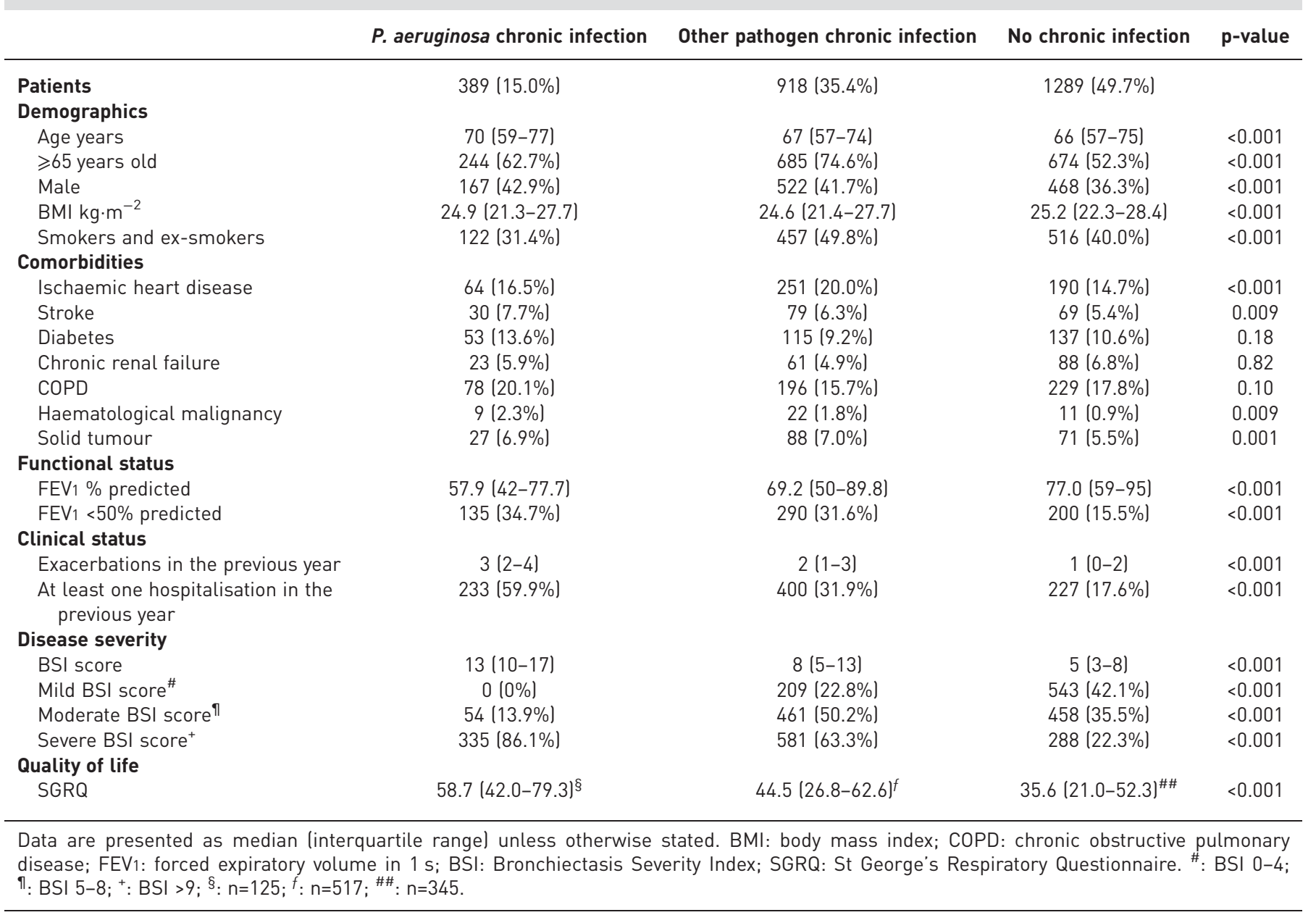

A univariate analysis of the impact $P$. aeruginosa chronic infection had in terms of mortality showed a higher mortality in the $P$. aeruginosa group than in all the other patients (HR 2.02; 95\% CI 1.53-2.66; $\mathrm{p}<0.0001$ ) (figure 1).

A multivariate analysis was then performed with the variables that were determined to be clinically relevant affecting mortality (table 3 ). These results show that the presence of a $P$. aeruginosa chronic infection in isolation does not have an independent impact in terms of mortality (HR 0.98; 95\% CI 0.701.36; $\mathrm{p}=0.89$ ). Other variables however, such as age (HR 1.05; 95\% CI 1.04-1.07; $\mathrm{p}<0.001$ ), exacerbation frequency (HR (per exacerbation) 1.06; 95\% CI 1.01-1.12; p=0.02) and hospitalised exacerbations (HR 1.60; $95 \%$ CI 1.17-2.19; $\mathrm{p}=0.003$ ) had an independent contribution to a higher mortality.

We hypothesised that mortality associated with $P$. aeruginosa chronic infection may be exacerbation dependent. A separate analysis in terms of mortality was made dividing the study population into four categories: 1) P. aeruginosa chronic infection with less than two exacerbations per year; 2) P. aeruginosa chronic infection with two or more exacerbations per year; 3) no P. aeruginosa and less than two exacerbations per year; 4) no $P$. aeruginosa and two or more exacerbations per year.

Patients with P. aeruginosa and two or more exacerbations per year had a higher mortality rate (20.5\%), followed by patients with frequent exacerbations without $P$. aeruginosa (12.5\%). In those with fewer than two exacerbations per year, the mortality rate in the presence of $P$. aeruginosa chronic infection was $9.7 \%$ versus $6.7 \%$ in those without $P$. aeruginosa chronic infection. Of note, only 62 out of 389 patients with $P$. aeruginosa had fewer than two exacerbations per year.

Survival curves of the four groups are presented in figure 2.

A multivariate analysis was performed using the same variables. Having $P$. aeruginosa chronic infection with fewer than two exacerbations had no impact in terms of mortality (HR 0.90 ; 95\% CI $0.39-2.12$; 
FIGURE 1 Kaplan-Meier log-rank test survival curve and univariate analysis for mortality: chronic Pseudomonas aeruginosa (PA) infection versus all other patients. Cox proportional hazard regression analysis. Hazard ratio for death for chronic PA infection $2.02195 \% \mathrm{Cl}$ $1.53-2.66, p<0.001)$.

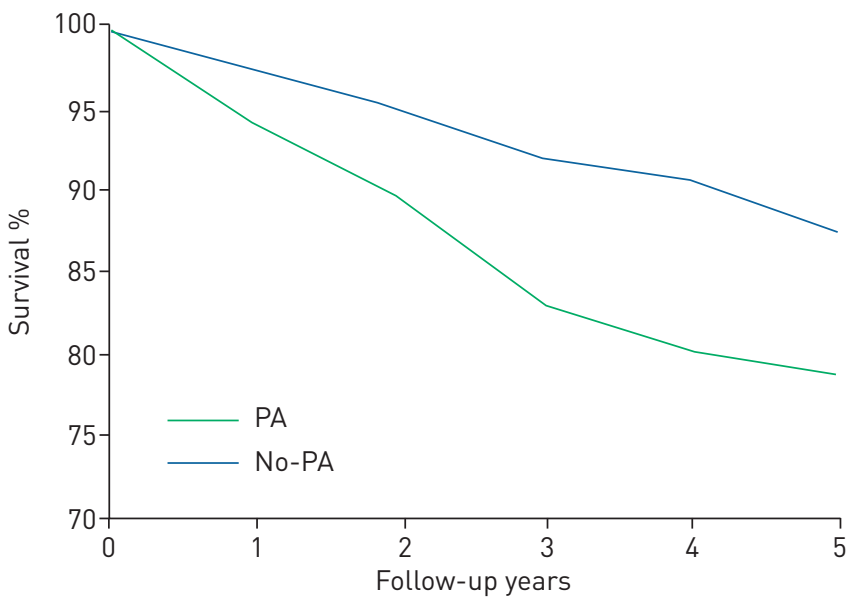

$\mathrm{p}=0.81$ ). Patients having two or more exacerbations per year had increased mortality (HR 1.74; 95\% CI $1.28-2.38 ; \mathrm{p}<0.001)$ an effect that was magnified in the presence of $P$. aeruginosa (HR 2.03; 95\% CI $1.36-$ $3.03 ; \mathrm{p}=0.001$ ).

A sensitivity analysis accounting for the centre as an independent variable in the analysis did not affect the conclusions (HR 1.09, 95\% CI $0.78-1.53$; p=0.6) in the fully adjusted analysis. The same was observed in the analysis of subgroups by exacerbation history: $P$. aeruginosa plus a history of two exacerbations per year (HR 2.22, 95\% CI 1.44-3.43, p<0.0001), two or more exacerbations per year without P. aeruginosa (HR 1.88, 95\% CI 1.34-2.60, p<0.0001). P. aeruginosa without a history of frequent exacerbations was not associated with mortality (HR $0.90,95 \%$ CI $0.38-2.12, \mathrm{p}=0.8$ ). Finally, in a sensitivity analysis including changes in treatment during follow-up as a time varying covariate, no differences in the conclusions were found. HRs were P. aeruginosa plus a history of two exacerbations per year (HR 2.01, 95\% CI 1.26-3.22,

\section{TABLE 3 Multivariate Cox regression analysis of factors associated with survival}

\begin{tabular}{lcc} 
Variable & Hazard ratio for death (95\% CI) & p-value \\
\hline Chronic Pseudomonas aeruginosa infection & $0.98(0.70-1.36)$ & 0.89 \\
Age years & $1.05(1.04-1.07)$ & $<.001$ \\
Male sex versus female & $1.29(0.99-1.67)$ & 0.06 \\
MRC dyspnoea score & $1.25(1.11-1.40)$ & $<0.001$ \\
FEV $\%$ predicted & & NA \\
$\quad<30$ & $1.00($ ref.) & 0.05 \\
$30-49$ & $0.66(0.43-0.99)$ & 0.001 \\
$50-79$ & $0.44(0.29-0.68)$ & 0.001 \\
$\geqslant 80$ & $0.45(0.28-0.73)$ & 0.003 \\
Hospitalised exacerbations & $1.60(1.17-2.19)$ & 0.17 \\
Radiology score ${ }^{\#}$ & $1.02(0.99-1.06)$ & 0.01 \\
BMI <18.5 kg-m ${ }^{-2}$ & $1.70(1.14-2.54)$ & 0.37 \\
Smoking status & $1.14(0.86-1.50)$ & 0.02 \\
Exacerbations in the previous year per additional & $1.06(1.01-1.12)$ & \\
$\quad$ exacerbation & & \\
Chronic Haemophilus influenzae infection & $0.59(0.40-0.86)$ & 0.007 \\
Chronic Staphylococcus aureus infection & $1.69(1.10-2.60)$ & 0.30 \\
Chronic Moraxella catarrhalis infection & $0.72(0.39-1.33)$ & 0.16 \\
Chronic Enterobacteriaceae infection & $0.71(0.44-1.15)$ & 0.03 \\
COPD & $1.41(1.04-1.90)$ & 0.14 \\
Diabetes & $1.27(0.92-1.75)$ & 0.03 \\
Chronic renal failure & $1.46(1.03-2.06)$ & 0.006 \\
Ischaemic heart disease & $1.47(1.12-1.93)$ & 0.46 \\
Oral long-term antibiotic suppressive therapy & $1.11(0.84-1.47)$ & 0.33 \\
Nebulised long-term antibiotic suppressive therapy & $0.80(0.51-1.25)$ & \\
\hline
\end{tabular}

MRC: Medical Research Council; FEV1: forced expiratory volume in $1 \mathrm{~s}$; BMI: body mass index; COPD: chronic obstructive pulmonary disease; NA: not applicable. ${ }^{\#}$ : Reiff score. 
FIGURE 2 Kaplan-Meier log-rank test survival curve: comparison between four groups: chronic Pseudomonas aeruginosa (PA) infection with fewer than two exacerbations per year; chronic PA infection with two or more exacerbations per year; no PA and fewer than two exacerbations per year; and no PA and two or more exacerbations per year.

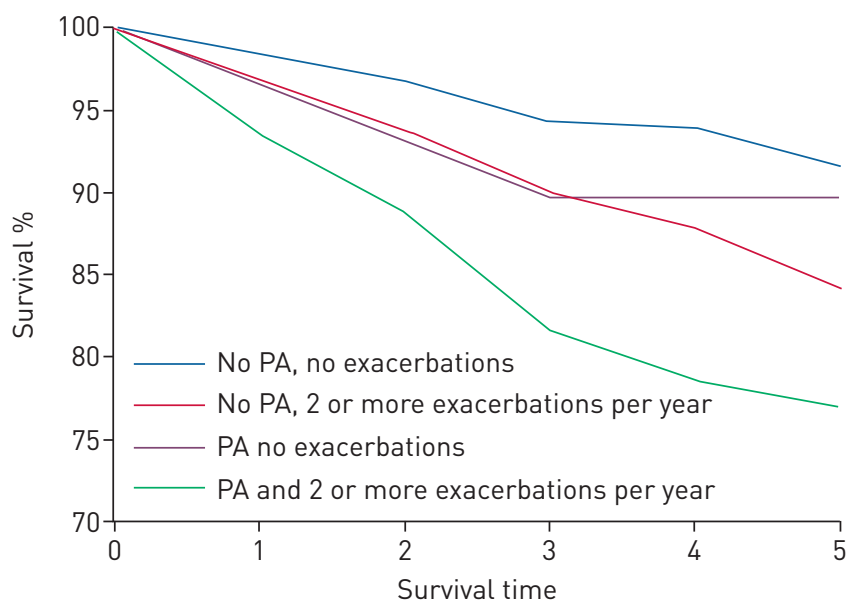

$\mathrm{p}=0.003$ ), two or more exacerbations per year without $P$. aeruginosa (HR 1.80, 95\% CI 1.27-2.55, $\mathrm{p}=0.001)$. $P$. aeruginosa without a history of frequent exacerbations was not associated with mortality (HR 0.82, 95\% CI $0.32-2.09, \mathrm{p}=0.7$ ). New antibiotic treatment (oral or inhaled) during follow-up was not significantly associated with mortality HR $0.78,95 \%$ CI $0.46-1.34, \mathrm{p}=0.4$ (table 4 ).

\begin{tabular}{|c|c|c|}
\hline Variable & Hazard ratio for death $(95 \% \mathrm{CI})$ & p-value \\
\hline \multicolumn{3}{|l|}{ Groups } \\
\hline $\begin{array}{l}\text { No chronic Pseudomonas aeruginosa infection with }<2 \\
\text { exacerbations per year }\end{array}$ & 1.00 (ref.) & NA \\
\hline $\begin{array}{l}\text { No chronic Pseudomonas aeruginosa infection with } \geqslant 2 \\
\text { exacerbations per year }\end{array}$ & $1.74(1.28-2.38)$ & $<0.001$ \\
\hline $\begin{array}{l}\text { Chronic Pseudomonas aeruginosa infection with <2 } \\
\text { exacerbations per year }\end{array}$ & $0.90(0.39-2.12)$ & 0.81 \\
\hline $\begin{array}{l}\text { Chronic Pseudomonas aeruginosa infection with } \geqslant 2 \\
\text { exacerbations per year }\end{array}$ & $2.03(1.36-3.03)$ & 0.001 \\
\hline Age years & $1.05(1.04-1.07)$ & $<0.001$ \\
\hline Male sex versus female & $1.29(1.00-1.68)$ & 0.055 \\
\hline Radiology score ${ }^{\#}$ & $1.03(0.99-1.06)$ & 0.11 \\
\hline $\mathrm{BMI}<18.5 \mathrm{~kg} \cdot \mathrm{m}^{-2}$ & $1.68(1.13-2.50)$ & 0.011 \\
\hline Smoking status & $1.11(0.84-1.47)$ & 0.45 \\
\hline MRC dyspnoea score & $1.24(1.16-1.45)$ & $<0.001$ \\
\hline \multicolumn{3}{|l|}{ FEV $1 \%$ predicted } \\
\hline$<30$ & 1.00 (ref.) & NA \\
\hline $30-49$ & $0.66(0.44-1.00)$ & 0.048 \\
\hline $50-79$ & $0.41(0.27-0.62)$ & $<0.001$ \\
\hline$\geqslant 80$ & $0.43(0.27-0.68)$ & $<0.001$ \\
\hline Chronic Haemophilus influenzae infection & $0.57(0.39-0.84)$ & 0.005 \\
\hline Chronic Staphylococcus aureus infection & $1.73(1.10-2.60)$ & 0.01 \\
\hline Chronic Moraxella catarrhalis infection & $0.78(0.43-1.44)$ & 0.43 \\
\hline Chronic Enterobacteriaceae infection & $0.78(0.49-1.24)$ & 0.29 \\
\hline COPD & $1.33(0.98-1.81)$ & 0.07 \\
\hline Diabetes & $1.28(0.93-1.75)$ & 0.13 \\
\hline Chronic renal failure & $1.58(1.13-2.23)$ & 0.008 \\
\hline Ischemic heart disease & $1.46(1.11-1.92)$ & 0.007 \\
\hline Oral long-term antibiotic suppressive therapy & $1.14(0.86-1.49)$ & 0.36 \\
\hline Nebulised long-term antibiotic suppressive therapy & $0.79(0.51-1.25)$ & 0.32 \\
\hline
\end{tabular}

BMI: body mass index; MRC: Medical Research Council; FEV1: forced expiratory volume in $1 \mathrm{~s}$; COPD: chronic obstructive pulmonary disease; NA: not applicable. ${ }^{\#}$ : Reiff score. 
Risk of exacerbations and hospital admissions

$P$. aeruginosa and $H$. influenzae chronic infection constituted predictors of future exacerbations: incident rate ratio (95\% CI) $1.14(1.04-1.27)$ and $1.13(1.03-1.24)$ respectively.

Patients with $P$. aeruginosa chronic infection had a much higher probability of having a hospital admission on a univariate analysis (odds ratio (OR): 4.96; 95\% CI 3.96-6.22; $\mathrm{p}<0.001$ ). This effect was also seen, although to a lesser degree, in a multivariable analysis (OR 2.28; 95\% CI 1.69-3.08; $\mathrm{p}<0.001$ ).

\section{Quality of life analysis}

The median value of SGRQ in the $P$. aeruginosa group was 58.1 points (IQR-54.0-62.2) and was higher than the median values for the other pathogens and the no chronic infection groups. A multivariable linear regression was performed showing an independent increase in the SGRQ of 7.46 points (95\% CI 2.93-12.00; $\mathrm{p}=0.001$ ), higher than the minimal clinically important difference (MCID) for SGRQ, which is 4 points [22]. These results were confirmed on the generalised linear model with quasibinomial errors and a logit link.

\section{Discussion}

This is the largest single study of bronchiectasis patients with the aim to assess the prevalence and burden of $P$. aeruginosa chronic infection.

While multiple studies have reported an association between $P$. aeruginosa and more severe bronchiectasis, and some have shown an association with poorer clinical outcomes, these were predominantly single centre, with small samples sizes and could not provide any information as to whether P. aeruginosa was a cause of poor outcomes or simply a marker of more severe disease. Our study contributes to this debate by clearly demonstrating that after adjustment for multiple possible confounding variables, $P$. aeruginosa remains associated with increased exacerbations, increased hospital admission risk and worse QoL. An association with mortality was only demonstrated in patients experiencing frequent exacerbations.

The prevalence of $P$. aeruginosa chronic infection in this study population was $15.0 \%$, which is in keeping with the ranges of prior publications (9-31\%) [12-17]. In a recent meta-analysis, FINCH et al. identified a rate of $P$. aeruginosa colonisation of $24.1 \%$, but the various studies included had different definitions of chronic colonisation, with some regarding a single isolate as indicative of chronic infection [11]. Mortality in this study population, with a follow-up of 5 years, was $10.8 \%$, which is within the range of prior quoted studies over 4-5 years follow-up (Loebinger et al. [12] 9\% at 4 years, Goeminne et al. [23] 20\% at 5 years).

Several studies have identified $P$. aeruginosa chronic infection as a risk factor for mortality in bronchiectasis patients $[11,12,21,23,24]$. A pooled OR for mortality was 2.95 (95\% CI 1.98-4.40; $\mathrm{p}<0.0001)$ in the Finch meta-analysis. In our cohort, for the univariate analysis we had a HR of $2.02(95 \%$ CI 1.53-2.66; $\mathrm{p}<0.0001)$. Because this group of patients has more risk factors than other groups, such as poorer lung function, increased age, certain comorbidities, it is important to clearly assess if $P$. aeruginosa really has an independent impact factor on mortality. In that matter, a multivariate analysis was performed. Herein independent predictors of mortality were age, low BMI, number of exacerbations, but in isolation, persistent infection with $P$. aeruginosa was not.

To better understand why there was such a difference between univariate and multivariate analysis, the study population was divided into four groups (in terms of $P$. aeruginosa chronic infection and number of exacerbations) and a new multivariate analysis was performed. It became clear that having exacerbations plays a major role in mortality, independent of $P$. aeruginosa status. These data strengthen the importance of reducing exacerbations in all bronchiectasis patients, particularly in the case of $P$. aeruginosa chronic infection, where a higher mortality risk is seen with frequent exacerbations.

Even allowing for varying thresholds for admission across different healthcare systems, where home intravenous antibiotic may or may not be available, $P$. aeruginosa appears strongly correlated with exacerbation frequency and the risk of hospitalisation. The risk of hospital admission is related to severity of disease, number of exacerbations and the antibiotic susceptibility pattern of the pathogen responsible for the exacerbation, which in the case of $P$. aeruginosa is often limited to intravenous antibiotics.

QoL was evaluated in this study using the SGRQ, which has been extensively validated in bronchiectasis patients [25]. P. aeruginosa chronic infection has been associated with a lower QoL, and in the FINCH et al. [11] meta-analysis there was an 18.2 points decrement in the SGRQ [21, 26]. The decrement justified by the presence of $P$. aeruginosa chronic infection was of 7.46 points $(95 \%$ CI $2.93-12.00 ; \mathrm{p}=0.001)$ which is considerably lower than the meta-analysis result. However, since it was adjusted for multiple variables, it gives us a better understanding of the real impact of this chronic infection in QoL, and it is still clearly clinically significant and far above the MCID for SGRQ. 
If we accept now that there is robust evidence that $P$. aeruginosa drives worse outcomes in bronchiectasis there are a number of clinical implications. First, a limited amount of observational data suggest that it may be possible to prevent $P$. aeruginosa chronic infection by giving eradication treatment with oral, intravenous and/or inhaled antibiotics in combination [27]. A conditional recommendation in favour of this approach was provided in the 2017 ERS bronchiectasis guidelines [28]. Secondly, if P. aeruginosa infection cannot be prevented, then our data suggest that patients experiencing frequent exacerbations have the worst outcomes and so exacerbation prevention should be the key therapeutic focus. Long-term macrolides have been shown to reduce exacerbation frequency in bronchiectasis, but these studies included few subjects with $P$. aeruginosa [29]. Inhaled antibiotics such as colistin, aminoglycosides and aztreonam have been tested in large populations of patients with $P$. aeruginosa with equivocal results [28]. Larger studies are awaited. The ERS bronchiectasis guidelines provided a conditional recommendation for the use of inhaled antibiotics for patients with $P$. aeruginosa and three or more exacerbations per year, with macrolides to be added if inhaled antibiotics fail to control exacerbations [28]. Our results reinforce the importance of following these recommendations alongside optimising airway clearance, vaccination and the use of pulmonary rehabilitation in these patients [28].

The strengths of this study are the substantial number of patients included, the evaluation at different bronchiectasis clinical sites in different countries across Europe, and the considerable amount of patient data collected. A significant number of events, related to the large study population, enabled us to have a robust multivariate analysis with many variables, which helped to better understand the independent impact of $P$. aeruginosa chronic infection. Despite this, we acknowledge that the number of patients with $P$. aeruginosa not having frequent exacerbations was low. We cannot exclude the possibility that this group has an increased risk of death.

The main limitation of this study is not having a description of the cause of death in all cohorts. It would have been useful to know the number of deaths associated with respiratory causes. Another important limitation is that $P$. aeruginosa diagnosis is based on sputum culture, which is known to be insensitive, and the methods and frequency of sputum culture are not standardised [30]. This could potentially underestimate the frequency of $P$. aeruginosa chronic infection. Another limitation is the absence of an analysis of the impact that the $P$. aeruginosa phenotype/strain type and resistance pattern could have in terms of the patient's outcomes [30].

This study gives us a more accurate view of the real $P$. aeruginosa chronic infection prevalence across Europe. It also enables us to better understand its burden of disease, with a connection to poorer lung function, more exacerbations and hospitalisations, and a worse QoL. In terms of mortality, the relationship between $P$. aeruginosa and an increased risk of death, described previously in the literature, seems to be closely related with the fact that these patients have more exacerbations and more hospitalisations, rather than just the simple fact of being chronically infected by this pathogen. Our analysis cannot fully account for the "chicken or egg" question of which characteristic comes first in disease. It is not known whether patients with $P$. aeruginosa have more exacerbations and therefore have worse outcomes, or whether frequently exacerbating patients have a poor prognosis with frequent antibiotic courses leading to $P$. aeruginosa infection and poor outcomes. Our study can only demonstrate that the combination of these two parameters increases mortality. The mechanism by which exacerbations contribute to increase mortality in bronchiectasis is not fully understood and should be the subject of detailed translational research. Our work could have major implications for healthcare policy; it implies that adverse effects of $P$. aeruginosa may be mitigated by exacerbation prevention. Furthermore, it highlights the important impact of exacerbations in the non-Pseudomonas population, validating this as a key therapeutic target.

\section{References}

1 McShane PJ, Naureckas ET, Tino G, et al. Non-cystic fibrosis bronchiectasis. Am J Respir Crit Care Med 2013; 188: 647.

2 Chalmers JD, Smith MP, McHugh BJ, et al. Short- and long-term antibiotic treatment reduces airway and systemic inflammation in non-cystic fibrosis bronchiectasis. Am J Respir Crit Care Med 2012; 186: 657-665. Cole PJ. Inflammation: a two-edged sword-the model of bronchiectasis. Eur J Respir Dis Suppl 1986; 147: 6-15. Whitters D, Stockley R. Immunity and bacterial colonisation in bronchiectasis. Thorax 2012; 67: 1006-1013. Cullen L, McClean S. Bacterial adaptation during chronic respiratory infection. Pathogens 2015; 4: 66-89. Chalmers JD, Hill AT. Mechanisms of immune dysfunction and bacterial persistence in non-cystic fibrosis bronchiectasis. Mol Immunol 2013; 55: 27-34.

7 Emerson J, Rosenfeld M, McNamara S, et al. Pseudomonas aeruginosa and other predictors of mortality and morbidity in young children with cystic fibrosis. Pediatr Pulmonol 2002; 34: 91-100.

8 Aliberti S, Lonni S, Dore S, et al. Clinical phenotypes in adult patients with bronchiectasis. Eur Respir J 2016; 47: 1113-1122.

9 Evans SA, Turner SM, Bosch BJ, et al. Lung function in bronchiectasis: the influence of Pseudomonas aeruginosa. Eur Respir J 1996; 9: 1601-1604. 
10 Davies G, Wells AU, Doffman S, et al. The effect of Pseudomonas aeruginosa on pulmonary function in patients with bronchiectasis. Eur Respir J 2006; 28: 974-979.

11 Finch S, McDonnell MJ, Abo-Leyah $\mathrm{H}$, et al. a comprehensive analysis of the impact of Pseudomonas aeruginosa colonization on prognosis in adult bronchiectasis. Ann Am Thorac Soc 2015; 12: 1602-1611.

12 Loebinger MR, Wells AU, Hansell DM, et al. Mortality in bronchiectasis: a long-term study assessing the factors influencing survival. Eur Respir J 2009; 34: 843-849.

13 Pasteur MC, Helliwell SM, Houghton SJ, et al. An investigation into causative factors in patients with bronchiectasis. Am J Respir Crit Care Med 2000; 162: 1277-1284.

14 Martínez-Garcia MA, Soler-Cataluña JJ, Perpiña-Tordera M, et al. Factors associated with lung function decline in adult patients with stable non-cystic fibrosis bronchiectasis. Chest 2007; 132: 1565-1572.

15 King PT, Holdsworth SR, Freezer NJ, et al. Microbiologic follow-up study in adult bronchiectasis. Respir Med 2007; 101: 1633-1638.

16 Ho PL, Chan KN, Ip MSM, et al. The effect of Pseudomonas aeruginosa infection on clinical parameters in steady-state bronchiectasis. Chest 1998; 114: 1594-1598.

17 Suarez-Cuartin G, Smith A, Abo-Leyah H, et al. Anti-Pseudomonas aeruginosa IgG antibodies and chronic airway infection in bronchiectasis. Respir Med 2017; 128: 1-6.

18 McDonnell MJ, Jary HR, Perry A, et al. Non cystic fibrosis bronchiectasis: a longitudinal retrospective observational cohort study of Pseudomonas persistence and resistance. Respir Med 2015; 109: 716-726.

19 Pasteur MC, Bilton D, Hill AT, et al. British Thoracic Society guideline for non-CF bronchiectasis. Thorax 2010; 65: Suppl. 1, i1-i58.

20 Clinical and Laboratory Standards Institute (CLSI). Performance standards for antimicrobial susceptibility testing: 14th informational supplement [CLSI document M100-S14]. Wayne, Clinical and Laboratory Standards Institute, 2004.

21 Chalmers JD, Goeminne P, Aliberti S, et al. The Bronchiectasis Severity Index: an international derivation and validation study. Am J Respir Crit Care Med 2014; 189: 576-585.

22 Wilson CB, Jones PW, O'Leary CJ, et al. Validation of the St. George's Respiratory Questionnaire in bronchiectasis. Am J Respir Crit Care Med 1997; 156: 536-541.

23 Goeminne PC, Nawrot TS, Ruttens D, et al. Mortality in non-cystic fibrosis bronchiectasis: a prospective cohort analysis. Respir Med 2014; 108: 287-296.

24 Martínez-García MA, de Gracia J, Vendrell Relat M, et al. Multidimensional approach to non-cystic fibrosis bronchiectasis: the FACED score. Eur Respir J 2014; 43: 1357-1367.

25 Spinou A, Fragkos KC, Lee KK, et al. The validity of health-related quality of life questionnaires in bronchiectasis: a systematic review and meta-analysis. Thorax 2016; 71: 683-694.

26 Wilson CB, Jones PW, O'Leary CJ, et al. Effect of sputum bacteriology on the quality of life of patients with bronchiectasis. Eur Respir J 1997; 10: 1754-1760.

27 Vallières E, Tumelty K, Tunney MM, et al. Efficacy of Pseudomonas aeruginosa eradication regimens in bronchiectasis. Eur Respir J 2017; 49: 1600851.

28 Polverino E, Goeminne PC, McDonnell MJ, et al. European Respiratory Society Guidelines for the management of adult bronchiectasis. Eur Respir J 2017; 50: 1700629.

29 Wong C, Jayaram L, Karalus N, et al. Azithromycin for prevention of exacerbations in non-cystic fibrosis bronchiectasis (EMBRACE): a randomised double blind placebo-controlled trial. Lancet 2012; 380: 660-667.

30 Hilliam Y, Moore MP, Lamont IL, et al. Pseudomonas aeruginosa adaptation and diversification in the non-cystic fibrosis bronchiectasis lung. Eur Respir J 2017; 49: 1602108. 